\title{
BIOLOGIA REPRODUTIVA DE ASPIDORAS FUSCOGUTTATUS (SILURIFORMES, CALLICHTHYIDAE) EM RIACHO DE CABECEIRA DA BACIA DO ALTO RIO PARANÁ
}

\author{
Renato Braz de Araujo ${ }^{1}$ \\ Valdener Garutti ${ }^{1,2}$
}

\begin{abstract}
REPRODUCTIVE BIOLOGY OF ASPIDORAS FUSCOGUTTATUS (SILURIFORMES, CALLICHTHYIDAE) IN A HEADSTREAM FROM UPPER PARANÁ RIVER BASIN. The reproductive biology of Aspidoras fuscoguttatus Nijssen \& Isbrücker, 1976 from a stream in São José do Rio Preto, northwestern São Paulo State, Brazil, was monthly investigated in the period of August 1999 to July 2000. Measurements of total length, body weight, gonadal weight and macroscopic assessment of gonadal maturation were performed. Environmental parameters were considered in order to verify associations with the reproductive period. Populational structure showed total length amplitude between 14.2 and $50.8 \mathrm{~mm}$. Pronounced sexual dimorphism was verified. The largest mean values of gonadosomatic relation for females coincided with the rainy season (November to March). Mean length at first sexual maturity was different for males $(30.5 \mathrm{~mm})$ and females $(37.1 \mathrm{~mm})$. Fecundity varied between 51 and 166 oocytes. Gonadal maturation curve, frequency of maturation stages and size frequency distributions of oocytes in mature ovaries revealed a long reproductive period, suggesting fractional spawning.
\end{abstract}

KEYWORDS. Biology, Aspidoras, Callichthyidae, Brazil.

\section{INTRODUÇÃO}

Na bacia do alto rio Paraná, associados a grandes rios, existe grande quantidade de riachos de cabeceiras habitados principalmente por peixes de pequeno porte (menores que 12 centímetros de comprimento padrão), dependentes da vegetação marginal para alimentação, refúgio e reprodução. Uma das famílias com representantes nesses ambientes é Callichthyidae (BuCKUP, 1999), que possui aproximadamente 165 espécies, 8 gêneros e 2 subfamílias: Callichthyinae (Callichthys Scopoli, 1877; Dianema Cope, 1871; Hoplosternum Eigenmann \& Eigenmann, 1888; Lepthoplosternum Reis, 1997; Megalechis Reis, 1997) e Corydoradinae (Aspidoras Ihering, 1907; Corydoras Lacépède, 1803; Brochis Cope, 1872) (ReIs, 1998).

1. Programa de Pós-Graduação em Aqüicultura, Centro de Aqüicultura, Universidade Estadual Paulista, Rod. Carlos Tomanni, Km 5, 14870-000, Jaboticabal, SP, Brasil. (renatoaraujo@zipmail.com.br)

2. Depto de Zoologia e Botânica, Instituto de Biociências, Letras e Ciências Exatas e Centro de Aqüicultura, UNESP, R. Cristovão Colombo, 2265 , 15054-000, São José do Rio Preto, SP, Brasil. 
Trabalhos sobre biologia de cascudos do gênero Aspidoras são escassos. As informações disponíveis praticamente restringem-se às espécies utilizadas em aquariofilia (Burgess, 1989) e, portanto, obtidas sob condições artificiais. Segundo BritTo (2000), a maioria das 17 espécies desse gênero ocorre em áreas restritas de algumas das maiores bacias hidrográficas brasileiras, sugerindo forte endemismo (NIJSSEN \& ISBRÜCKER, 1976). Aspidoras fuscoguttatus Nijssen \& Isbrücker, 1976 é um cascudo comum em riachos de cabeceiras da bacia do rio Preto, rio Grande, alto rio Paraná; é utilizado como peixe ornamental na região de São José do Rio Preto, São Paulo. Entretanto, nenhuma pesquisa sobre sua biologia reprodutiva foi realizada; na literatura, o único registro de $A$. fuscoguttatus é o de GARUTTI (1988), que analisou a distribuição longitudinal da ictiofauna em um córrego da bacia do alto rio Paraná.

Objetiva-se investigar a biologia reprodutiva, a estrutura populacional e a relação peso-comprimento de $A$. fuscoguttatus, procedente de um riacho afluente do córrego do Cedro, abordando-se proporção sexual, comprimento da primeira maturação gonadal, fecundidade, tipo de desova e época de reprodução. Avaliam-se ainda alguns dados ambientais e sua possível associação com o período reprodutivo.

\section{MATERIAL E MÉTODOS}

O estudo foi desenvolvido em um riacho de planalto (2052'06"S, $\left.49^{\circ} 16^{\prime} 40^{\prime \prime} \mathrm{W}\right)$, situado no distrito de Engenheiro Schmidt, município de São José do Rio Preto, região Noroeste do Estado de São Paulo. É um riacho de primeira ordem na classificação de STRAHLER (1957), com cerca de $540 \mathrm{~m}$ de extensão; é afluente do córrego do Cedro, que deságua no rio Preto, drenagem do rio Grande, bacia do alto rio Paraná.

Exemplares de A. fuscoguttatus foram coletados mensalmente em três pontos do riacho (nascente, curso médio e desembocadura), no período de agosto de 1999 a julho de 2000, exceto janeiro de 2000, sempre no período matutino, entre $7 \mathrm{~h} 20 \mathrm{~min}$ e $11 \mathrm{~h} 40 \mathrm{~min}$. A coleta foi realizada com peneira de malha fina $(0,3 \times 0,3 \mathrm{~mm})$, sendo os peixes acondicionados em caixa de isopor com gelo e transportados para análise em laboratório. Espécimes-testemunho foram depositados na Coleção de Peixes do Departamento de Zoologia e Botânica, UNESP, São José do Rio Preto (DZRP 4414-4434; 4436-4457).

De cada exemplar foram registradas as variáveis comprimento total $(\mathrm{Ct})$ em milímetros, peso total $(\mathrm{Pt})$ em gramas, sexo, estádio de maturação gonadal e peso das gônadas $(\mathrm{Pg})$ em gramas. Todos os exemplares foram analisados no mesmo dia da coleta, exceto 155 separados por amostragem estratificada a partir da definição de classes de comprimento total. Esse tipo de amostragem foi adotado visando incluir na análise indivíduos de diversos tamanhos e idades para melhor caracterização da população estudada. A estrutura populacional foi verificada por meio de histograma, com base na freqüência relativa das classes de comprimento total. Considerou-se indivíduos indiferenciados aqueles cujas gônadas não permitiram a distinção do sexo. A proporção sexual foi calculada utilizando-se o teste Qui-Quadrado (SOKAL \& ROHLF, 1981).

Calculou-se a relação peso-comprimento pela equação $\mathrm{Pt}=\mathrm{aCt}^{\mathrm{b}}$, em que $\mathrm{Pt}$ corresponde ao peso total, $\mathrm{Ct}$ comprimento total e a e b são estimativas dos parâmetros de regressão, cujos valores foram obtidos ajustando-se aos dados de logaritmo de peso total e comprimento total à expressão referida, pelo método dos mínimos quadrados. O comprimento médio da primeira maturação gonadal $\left(\mathrm{C}_{50}\right)$ foi estimado conforme VAZZOLER (1996).

A determinação do sexo e a identificação dos estádios de maturação gonadal foram realizadas sob estereomicroscópio, mediante incisão longitudinal do abdômen, com as gônadas expostas, retiradas e pesadas. Testículos imaturos são de tamanho reduzido, filiformes, esbranquiçados, visíveis a olho nu com o contraste obtido pelo uso de fixador (solução de Bouin); em maturação são desenvolvidos, achatados dorso-ventralmente, recortados e esbranquiçados; maduros são volumosos e, esgotados, flácidos, com aspecto hemorrágico. Ovários imaturos ocupam menos de $1 / 3$ da cavidade abdominal, com ovócitos não visíveis a olho nu; em maturação de $1 / 3$ a $2 / 3$, com ovócitos visíveis; maduros ocupam quase totalmente a cavidade, túrgidos, bastante vascularizados, com ovócitos visíveis, enquanto os esgotados 
menos de 1/3, com poucos ovócitos de diversos tamanhos.

Para a análise da fecundidade foram utilizadas 19 fêmeas, cujos ovários estavam no estádio maduro. Foram contados todos os ovócitos com diâmetro superior a 159 micrômetros, medida limite para sua identificação sob estereomicroscópio. Para tanto, as gônadas foram colocadas em solução de Gilson para dissociação dos ovócitos, sendo, posteriormente, conservadas em álcool a 70\% (VAZZOLER, 1981). O tipo de desova foi determinado pela análise da freqüência relativa das classes de diâmetro dos ovócitos (VAZZOLER, 1981).

A relação gonadossomática (RGS) foi determinada pela expressão $(\mathrm{Pg} / \mathrm{Pc})$ x 100 , em que $\mathrm{Pg}$ corresponde ao peso dos ovários ou testículos, em gramas e Pc ao peso do corpo, também em gramas. $\mathrm{O}$ período reprodutivo foi estabelecido por meio da distribuição mensal dos valores da relação gonadossomática e da freqüência relativa dos estádios de maturação gonadal.

Durante as coletas, dados físico-químicos da água foram obtidos nos três pontos do riacho (nascente, curso médio e desembocadura). No campo foram mensuradas temperatura da água e condutividade elétrica. Amostras de água foram coletadas para determinação, em laboratório, do oxigênio dissolvido, pH, turbidez e alcalinidade. Todas as análises foram efetuadas com apenas uma medição, exceto oxigênio, medido três vezes. Os valores mensais de precipitação pluviométrica foram obtidos junto ao Escritório de Desenvolvimento Rural da Coordenadoria de Assistência Técnica Integral (EDRCATI) do Estado de São Paulo, obtidos à cerca de $4 \mathrm{~km}$ do riacho. Visando verificar se há relação entre variáveis ambientais (temperatura da água, condutividade elétrica, oxigênio dissolvido, $\mathrm{pH}$, turbidez, alcalinidade e precipitação pluviométrica) e indicadores reprodutivos de A. fuscoguttatus (relação gonadossomática média para as fêmeas, número mensal de indivíduos maduros e fecundidade), aplicouse o coeficiente de correlação $\underline{\mathrm{r}}$ de Pearson (SoKal \& RoHLf, 1981) por meio do programa Statdisk 5.0. As abreviaturas no texto referem-se a: média (X), desvio padrão (DP) e tamanho da amostra (n).

\section{RESULTADOS E DISCUSSÃO}

No período de amostragem foi capturado um total de 544 exemplares de $A$. fuscoguttatus, sendo 70 machos, 110 fêmeas, 209 indiferenciados e 155 exemplares separados por amostragem estratificada. A classe modal de comprimento total foi 34-37 $\mathrm{mm}(16,91 \%)$ (fig. 1), próxima à constatada por GARUTTI (1988), que analisou 86 exemplares de A. fuscoguttatus de 20 a $55 \mathrm{~mm}$ provenientes da bacia do alto rio Paraná. NisSSEN \& ISBRÜCKER (1976), descrevendo a espécie, registraram tamanhos entre 19 e $38 \mathrm{~mm}$ de comprimento padrão, em 25 espécimes analisados. Neste estudo, realizado com base em uma amostra mais numerosa, a amplitude do comprimento total variou entre 14,2 e 50,8 $\mathrm{mm}$. Esses resultados podem ser devido aos diferentes tamanhos das amostras, equipamentos de coleta utilizados e diferenças populacionais. Vale ressaltar que os dados de NIJSSEN \& ISBRÜCKER (1976) foram obtidos da medida comprimento padrão, diferindo de GARUTTI (1988) e deste trabalho, que utilizaram comprimento total.

Analisando a distribuição mensal de machos e fêmeas verificou-se que não ocorreram diferenças estatisticamente significativas. Entretanto, para o período total a diferença foi significativa $\left(\chi^{2}=4,23 ; p<0,05\right)$, sendo encontrada a proporção de 1,57 fêmeas para um macho. No riacho aqui estudado, foi encontrada a proporção de $61,1 \%$ de fêmeas e $38,9 \%$ de machos, próxima a encontrada por ARANHA et al. (1993) para Corydoras gr. carlae ( $66 \%$ de fêmeas e $34 \%$ de machos), mas diferente da obtida por esses autores para $C$. aeneus (Gill, 1958) (35,5\% de fêmeas e 64,5\% de machos). Os dados agora reportados para A. fuscoguttatus não permitem avanços na discussão das diferentes freqüências de machos e fêmeas; não se observou qualquer padrão a respeito, nos três pontos de coleta e ao longo do ano.

O dimorfismo sexual secundário observado para A. fuscoguttatus relaciona-se ao tamanho do corpo, sendo as fêmeas maiores que os machos. A amplitude do comprimento 
total variou entre 22,6 e $42 \mathrm{~mm}(\mathrm{X}=35,7 ; \mathrm{DP}=3,3 ; \mathrm{n}=70)$ nos machos e entre $30,0 \mathrm{e}$ $50,8 \mathrm{~mm}(\mathrm{X}=36,7 ; \mathrm{DP}=8,3 ; \mathrm{n}=110)$ nas fêmeas. A massa corpórea dos machos variou entre 1,6 e $9,7 \mathrm{~g}(\mathrm{X}=6,3 ; \mathrm{DP}=1,5 ; \mathrm{n}=70)$ e das fêmeas entre 3,9 e $18,6 \mathrm{~g}(\mathrm{X}=10,4 ; \mathrm{DP}$ $=3,6 ; \mathrm{n}=110)$. Na família Callichthyidae, Aspidoras menezesi Nijssen \& Isbrücker, $1976 \mathrm{e}$ Corydoras paleatus (Jenyns, 1842) apresentam fêmeas maiores que machos (BURGESS, 1989), ao contrário de Hoplosternum littorale (Hancock, 1828), cujos machos possuem maior tamanho e exercem comportamento de proteção à prole (HostACHE \& MoL, 1998). De acordo com Lowe-McConNell (1999), o dimorfismo sexual de ocorrência mais freqüente entre os Characiformes e Siluriformes é o maior tamanho alcançado pelas fêmeas em relação aos machos.

A equação obtida para a relação entre peso total $(\mathrm{Pt})$ e comprimento total $(\mathrm{Ct})$ foi $\mathrm{Pt}=0,0001$.C $^{3,0447}$. Segundo Le CREN (1951), valores de $\mathrm{b}$ dessa relação variam de 2,5 a 4,0 para os peixes. A relação $\mathrm{Pt} / \mathrm{Ct}$ resultou em curva de potência (fig. 2) com valor de $\mathrm{b}=3,045$.

O tamanho médio da primeira maturação gonadal de A. fuscoguttatus (fig. 3) foi maior nas fêmeas (37,1 mm) que nos machos (30,5 mm). ArANHA et al. (1993) registraram $30 \mathrm{~mm}$ como sendo o comprimento padrão do menor indivíduo maduro de Corydoras gr. carlae e C. aeneus. BERviAn \& FontouRA (1994) observaram maior freqüência de fêmeas reprodutivas de Corydoras paleatus entre 4,5 e 5,0 cm, estabelecendo o tamanho da primeira maturação como $4,75 \mathrm{~cm}$ de comprimento padrão. Entretanto, eles consideraram o tamanho da primeira maturação como aquele em que a maior parte dos indivíduos de uma população efetivamente atinge a maturação gonadal.

A avaliação da fecundidade representa um subsídio na estimativa de estoques pesqueiros e produção de alevinos em piscicultura. Pode fornecer indicações sobre a estratégia de vida da espécie. A fecundidade individual em A. fuscoguttatus variou de 51 a 166 ovócitos para exemplares de comprimento total variando entre 37,5 e $46,8 \mathrm{~mm}$. Em Corydoradinae, informações sobre fecundidade são assinaladas para A. menezesi ( 50 a 60 ovócitos), Corydoras paleatus (250 a 400 ovócitos), C. hastatus Eigenmann \& Eigenmann, 1888 (30 a 60 ovócitos) e C. pygmaeus Knaack, 1966 ( 20 a 40 ovócitos), todas utilizadas em aquariofilia e, portanto, obtidas em condições artificiais (BURGESS, 1989). Em relação às espécies de loricarí́deos, A. fuscoguttatus apresenta valores relativamente baixos quando comparada a Loricariichthys anus (Valenciennes, 1840) (466 ovócitos, BRUSCHI et al., 1997) e Hypostomus affinis Lacépède, 1803 (1784 ovócitos, MAZzonI \& CARAMASCHI, 1997). De acordo com LAGLER et al. (1977), a fecundidade é inversamente proporcional ao grau de cuidados parentais. Em Callichthyidae cuidados com a prole foram registrados em Hoplosternum littorale, Callichthys callichthys (Linnaeus, 1758) e C. paleatus Valenciennes, 1840 (MACHADO-AlLISON \& ZARET, 1984). Embora o presente estudo registre baixa fecundidade, o comportamento de proteção à prole não foi observado.

A distribuição da freqüência relativa do diâmetro de ovócitos de 38 ovários maduros, que variou de 159 a 1749 micrômetros, revela modas de ovócitos em desenvolvimento em fases distintas (fig. 4), o que indica desova do tipo parcelada. A estimativa da fecundidade em peixes com desova parcelada, feita com base apenas em análises macroscópicas, torna-se complexa devido à dificuldade de se distinguir ovócitos de reserva (estoque) daqueles em desenvolvimento. NIKOLSKY (1963) ressaltou a contribuição da desova parcelada para o aumento do número de ovócitos a serem postos durante o período reprodutivo, possibilitando maior probabilidade de sobrevivência da espécie. 


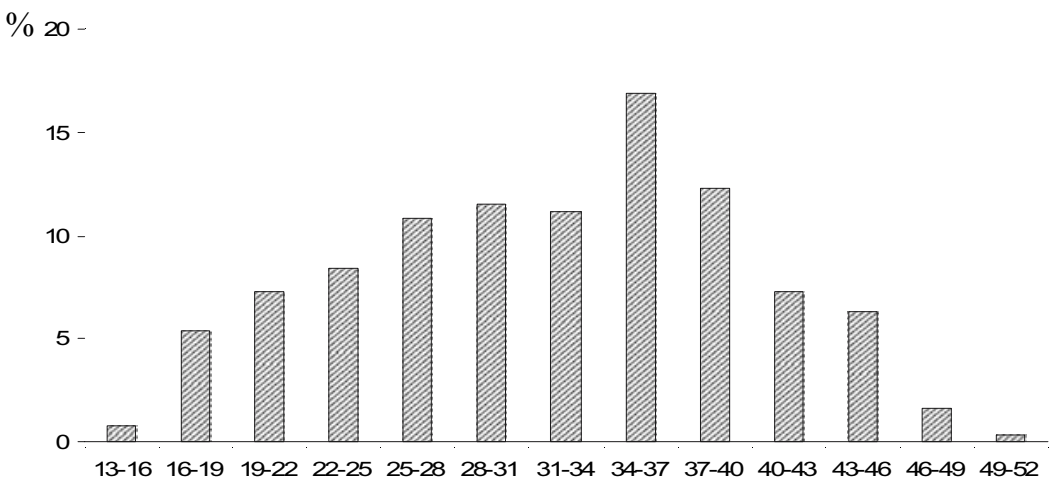

Classes de comprimento total (mm)

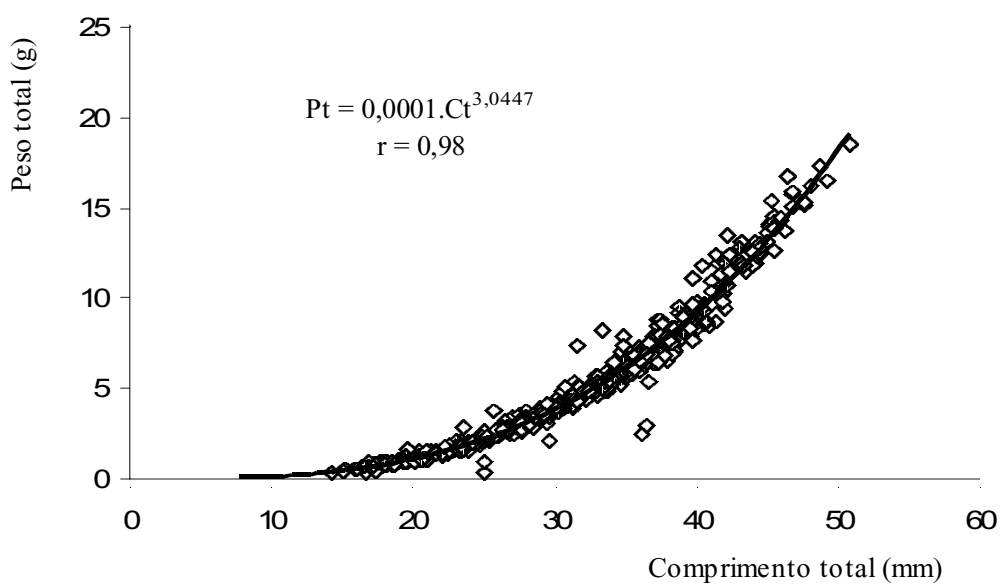

Figs. 1, 2. Aspidoras fuscoguttatus capturados em afluente do córrego do Cedro, São José do Rio Preto, São Paulo, de agosto/1999 a julho/2000. 1, distribuição da freqüência de classes de comprimento total; 2 , relação peso total $(\mathrm{Pt}) /$ comprimento total $(\mathrm{Ct})(\mathrm{r}$, coeficiente de correlação).

A distribuição mensal dos valores médios da relação gonadossomática de fêmeas (fig. 5) indica três picos (outubro, dezembro e março), dentro de um período de reprodução acentuada, agosto a março. A freqüência dos estádios de maturação gonadal (fig. 6), estabelecida a partir de observações macroscópicas dos ovários, indica atividade reprodutiva mais intensa no final do período chuvoso, fevereiro e março, mas fêmeas maduras ocorrem em todos os meses do ano. Constata-se, ainda, uma relação entre o longo período de atividade reprodutiva da espécie e a ocorrência de fêmeas imaturas em todos os meses, exceto setembro e novembro. ARANHA et al. (1993) encontraram fêmeas maduras de Corydoras gr. carlae durante todo o ano, enquanto C. aeneus não apresentou fêmeas em estado reprodutivo no período de março a junho. BERVIAN \& FonTOURA (1994), estudando a dinâmica populacional de C. paleatus, constataram reprodução praticamente 

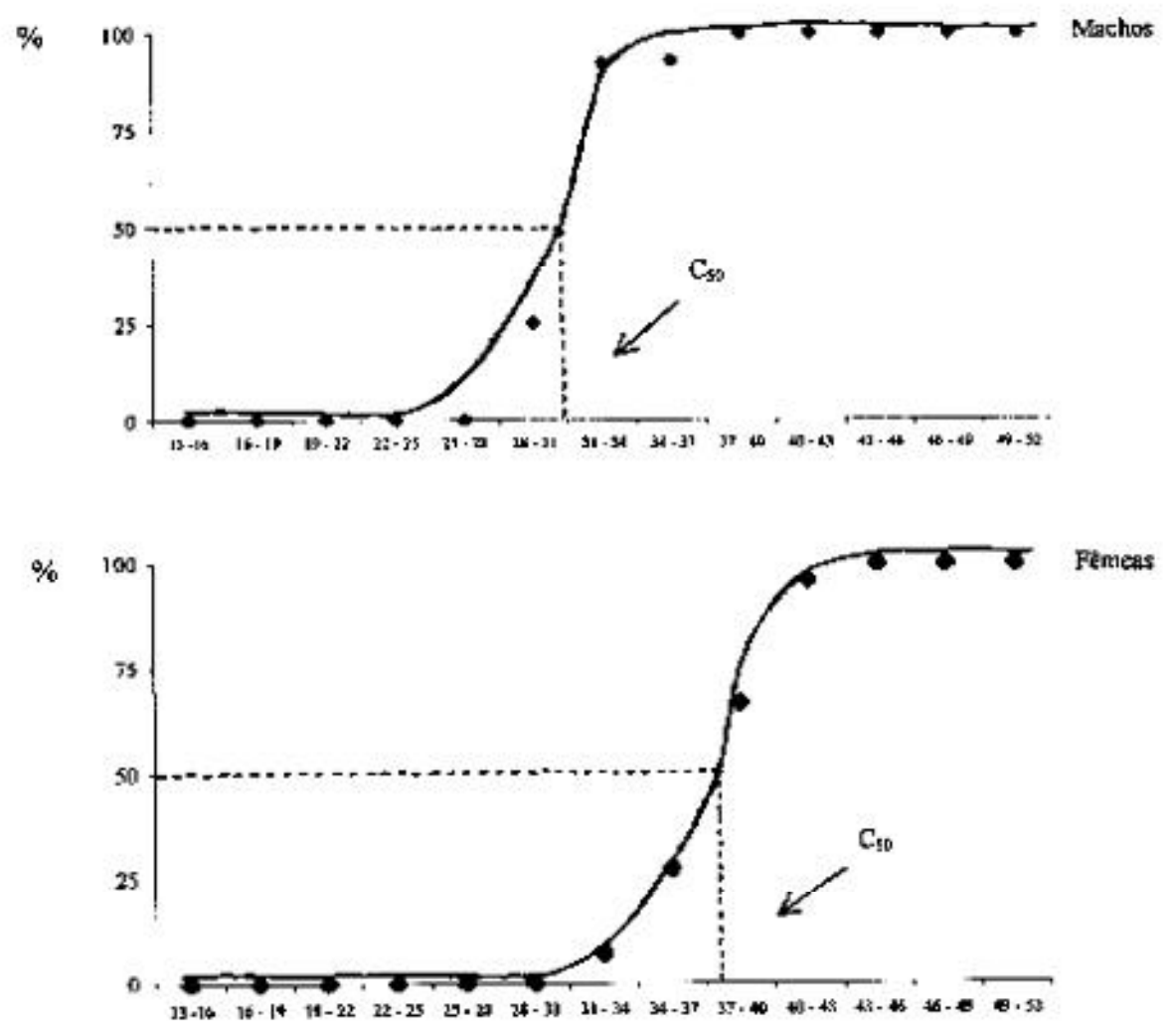

Clsoses de omporimedio lotal (mia)

Fig. 3. Aspidoras fuscoguttatus, comprimento médio da primeira maturação gonadal de machos e fêmeas capturados em afluente do córrego do Cedro, São José do Rio Preto, SP, de agosto/1999 a julho/ 2000 .

o ano todo, com um pico em fevereiro. O longo período de reprodução verificado em $A$. fuscoguttatus, Corydoras gr. carlae e C. paleatus corrobora a afirmação de NiKOLSKY (1963) de que espécies de regiões tropicais e subtropicais caracterizam-se por apresentar período reprodutivo longo. Segundo o referido autor, a probabilidade da espécie obter sucesso na reprodução será maior, pois a desova parcelada poderia evitar uma possível competição entre os jovens.

Para A. fuscoguttatus, do afluente do córrego do Cedro, a curva de maturação gonadal, a freqüência de ocorrência dos estádios de maturação gonadal e a distribuição da freqüência relativa dos diâmetros dos ovócitos de gônadas maduras sugerem que a 


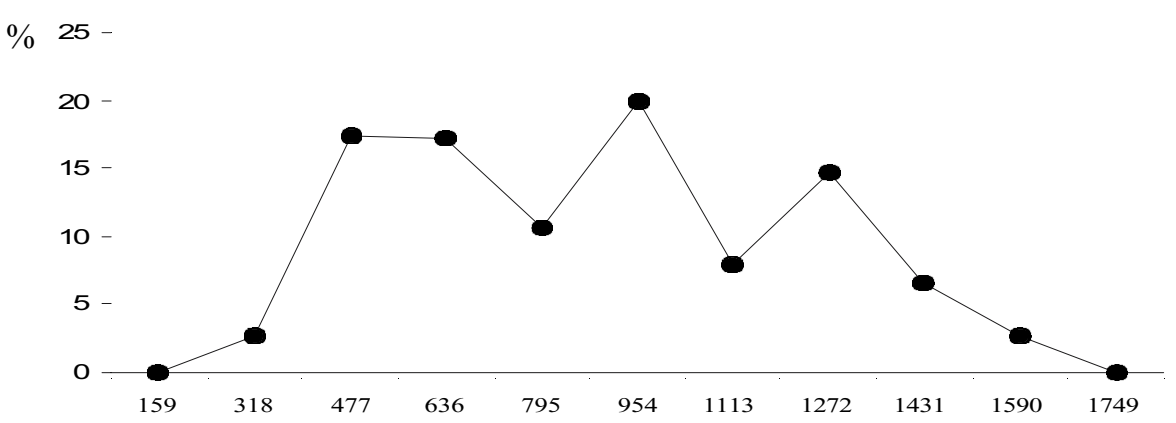

Classes de diâmetro $(\mu \mathrm{m})$

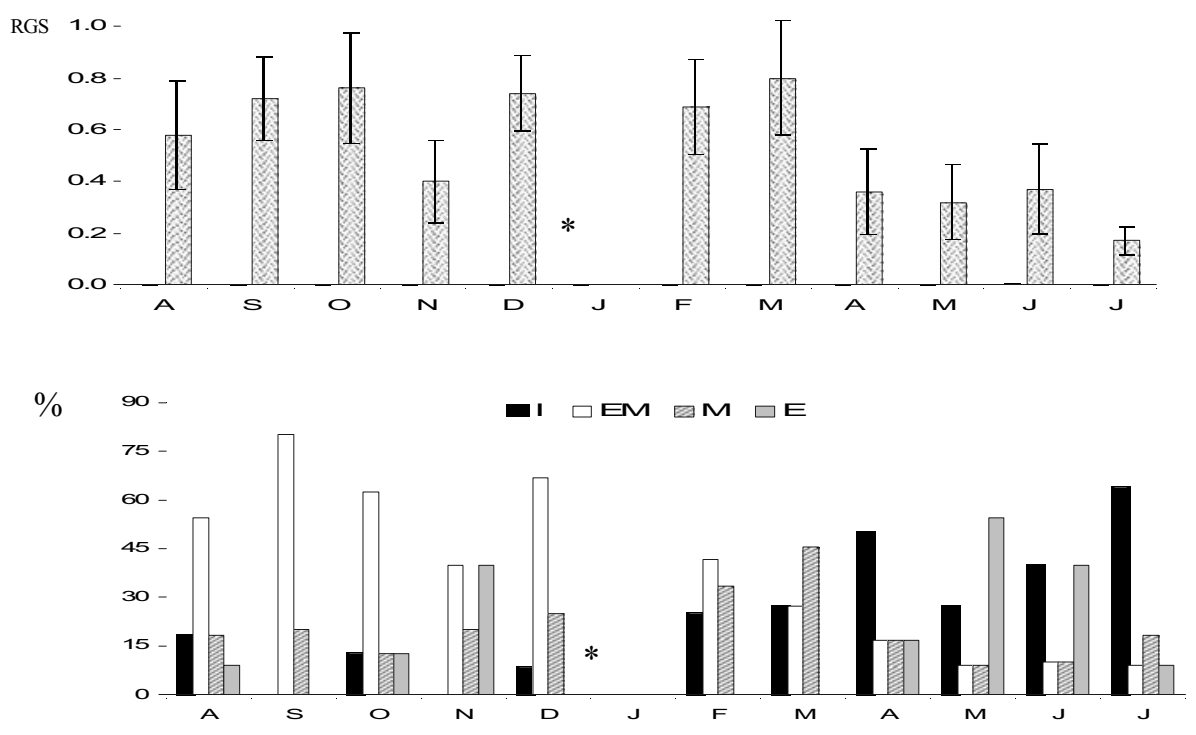

Figs. 4-6. Aspidoras fuscoguttatus capturados em afluente do córrego do Cedro, São José do Rio Preto, São Paulo, de agosto/1999 a julho/2000. 4, distribuição da freqüência relativa do diâmetro dos ovócitos de gônadas maduras; 5 , relação gonadossomática $(\mathrm{RGS})$ mensal de fêmeas $(\mathrm{n}=110)$ (média \pm erro padrão da média); 6 , freqüência mensal de fêmeas em cada estádio de maturação (E, esgotado; EM, em maturação; I, imaturo; M, maduro; *, não amostrado).

desova ocorre o ano todo e é do tipo parcelada.

Encontraram-se variações mensais de temperatura da água, condutividade elétrica, $\mathrm{pH}$, turbidez, alcalinidade, precipitação pluviométrica total e oxigênio dissolvido (fig. 7, tab. I). Na estação chuvosa (outubro a março), o riacho apresentou os maiores valores de temperatura, condutividade, turbidez, alcalinidade e precipitação, coincidindo com o período em que os valores médios da relação gonadossomática para as fêmeas de A. fuscoguttatus foram maiores. Apesar da RGS não apresentar correlação com a precipitação pluviométrica $(\mathrm{r}=0,05)$, os resultados aqui obtidos corroboram informações de AranHA et al. (1993) 
para Corydoras gr. carlae e C. aeneus e Alkins-Koo (2000) para C. aeneus de que os maiores valores da RGS das fêmeas ocorrem no período chuvoso. No presente estudo a RGS média das fêmeas foi positivamente correlacionada com a temperatura $(r=0,52)$ e turbidez $(\mathrm{r}=0,58)$. A fecundidade foi positivamente correlacionada com o oxigênio dissolvido $(\mathrm{r}=0,74)$ e com o $\mathrm{pH}(\mathrm{r}=0,45)$. Não se constatou correlação entre o número de indivíduos maduros e as variáveis ambientais analisadas. Entretanto, isto pode ser decorrente da pequena quantidade mensal de indivíduos maduros capturados, ou seja, de 1 a 6 para um total de 32 exemplares.
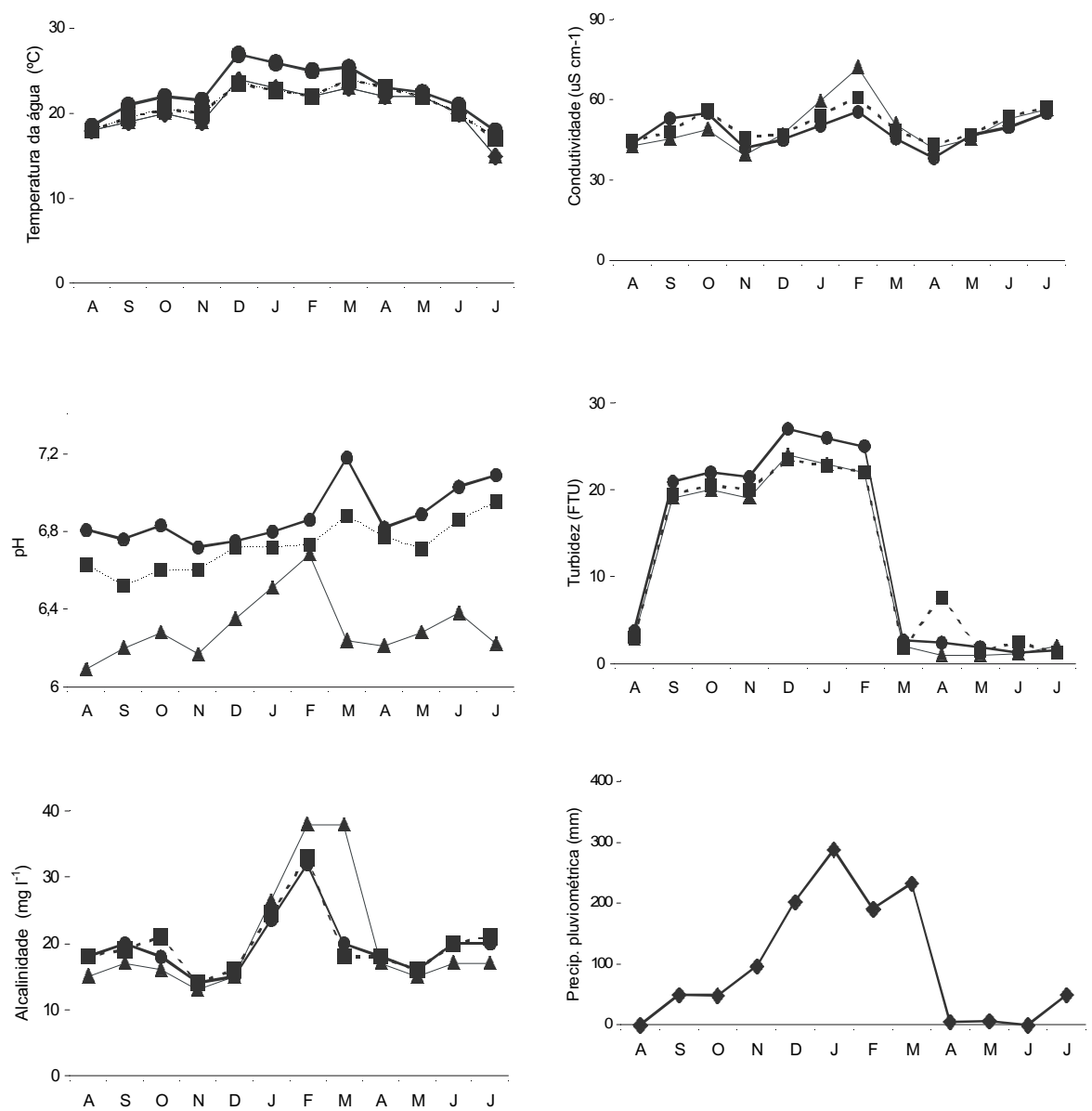

Fig. 7. Variação mensal de temperatura, condutividade elétrica, pH, turbidez, alcalinidade e precipitação pluviométrica em riacho afluente do córrego do Cedro, São José do Rio Preto, SP, de agosto/1999 a julho/2000 ( , nascente; , curso médio; , desembocadura). 
Tabela I. Média (X) e desvio padrão (DP) do oxigênio dissolvido ( $\left.m g \mathrm{l}^{-1}\right)$ amostrado em pontos do riacho afluente do córrego do Cedro, São José do Rio Preto, São Paulo, de agosto/1999 a julho/2000 (N, nascente; CM, curso médio; D, desembocadura).

\begin{tabular}{lrrr}
\hline \multirow{4}{*}{ Meses } & \multicolumn{4}{c}{$\mathrm{X} \pm \mathrm{DP}$} \\
\cline { 2 - 5 } & \multicolumn{4}{c}{$\mathrm{N}$} & \multicolumn{1}{c}{$\mathrm{CM}$} \\
\hline ago & $3,1 \pm 0,2$ & $8,8 \pm 0,5$ & $7,5 \pm 0,5$ \\
set & $3,5 \pm 0,3$ & $5,6 \pm 0,4$ & $4,8 \pm 1,5$ \\
out & $5,7 \pm 0,4$ & $10,1 \pm 0,6$ & $13,3 \pm 0,6$ \\
nov & $7,7 \pm 0,5$ & $13,5 \pm 0,1$ & $9,2 \pm 0,9$ \\
dez & $7,0 \pm 1,6$ & $14,9 \pm 0,3$ & $9,0 \pm 0,7$ \\
jan & $5,2 \pm 0,6$ & $14,3 \pm 0,5$ & $8,9 \pm 0,7$ \\
fev & $3,3 \pm 0,5$ & $13,6 \pm 0,7$ & $8,9 \pm 0,6$ \\
mar & $6,7 \pm 0,3$ & $7,0 \pm 0,4$ & $10,3 \pm 0,3$ \\
abr & $11,7 \pm 0,3$ & $7,0 \pm 0,4$ & $10,3 \pm 0,3$ \\
mai & $9,9 \pm 0,1$ & $7,8 \pm 0,2$ & $9,3 \pm 0,1$ \\
jun & $5,7 \pm 0,2$ & $12,7 \pm 0,1$ & $13,3 \pm 0,2$ \\
jul & $12,3 \pm 0,1$ & $11,2 \pm 0,2$ & $11,9 \pm 0,4$ \\
\hline
\end{tabular}

Agradecimentos. À CAPES pela concessão de bolsa ao autor sênior; aos Drs. Antonio F. M. Camargo e Virgínia S. Uieda, ambos da UNESP, pelas críticas e sugestões; ao Dr. Antonio J. Manzato (UNESP) pela orientação estatística; à professora Patrícia R. Delboni e ao matemático Gilson Heleno pelo auxílio na confecção das figuras 3 e 5, respectivamente; aos biólogos Henrique F. Chaves e Roselene S. C. Ferreira pela ajuda nos trabalhos de campo e laboratório.

\section{REFERÊNCIAS BIBLIOGRÁFICAS}

Alkins-Koo, M. 2000. Reproductive timing of fishes in a tropical intermittent stream. Env. Biol. Fishes, Dordrecht, 57(1):49-66.

Aranha, J. M. R.; Caramaschi, E. P. \& Caramaschi, U. 1993. Ocupação espacial, alimentação e época reprodutiva de duas espécies de Corydoras Lacépede (Siluroidei, Callichthyidae) coexistentes no rio Alambari (Botucatu, São Paulo). Revta bras. Zool., Curitiba, 10(3):453-466.

Bervian, G. \& Fontoura, N. F. 1994. Dinâmica populacional da ictiofauna da lagoa Fortaleza, Cidreira, Rio Grande do Sul. III. Corydoras paleatus (Jenyns, 1842) (Teleostei, Callichthyidae). Biociências, Porto Alegre, 2(2):15-23.

Britto, M. R. 2000. Aspidoras depinnai (Siluriformes: Callichthyidae): a new species from northeastern Brazil. Copeia, Gainesville, 2000:1048-1055.

Bruschi, W. JR.; Peret, A. C. et al. 1997. Reprodução de Loricariichthys anus (Valenciennes, 1840) da lagoa Emboaba, Osório, RS, Brasil. Revta bras. Biol., Rio de Janeiro, 57(4):677-685.

Buckup, P. A. 1999. Sistemática e biogeografia de peixes de riachos. In: Caramaschi, E. P.; Mazzoni, R. \& Peres-Neto, P. R. eds. Ecologia de peixes de riachos. Rio de Janeiro, PPGE-UFRJ. p.91-138.

BuRgess, W. E. 1989. An atlas of freshwater and marine catfishes: a preliminary survey of the Siluriformes. Neptune, TFH Publ. 784p.

GarutTI, V. 1988. Distribuição longitudinal da ictiofauna em um córrego da região noroeste do estado de São Paulo, bacia do rio Paraná. Revta bras. Biol., Rio de Janeiro, 48(4):747-759.

Hostache, G. \& Mol, J. H. 1998. Reproductive biology of the neotropical armoured catfish Hoplosternum littorale (Siluriformes, Callicthyidae): a synthesis stressing the role of the floating bubble nest. Aquat. Living Resourc., Montrouge, 11(3):173-185.

Lagler, K. F.; Bardach, J. E. \& Miller, R. R. 1977. Ichthyology. New York, Wiley. 506p.

LE CREN, E. D. 1951. The length-weight relationship and seasonal cycle in gonad weight and condition in the perch (Perca fluviatilis). J. Anim. Ecol., Oxford, 20(2):201-219.

Lowe-McConnell, R. H. 1999. Estudos ecológicos de comunidades de peixes tropicais. São Paulo, EDUSP. 534p. 
Machado-Allison, A. J. \& Zaret, T. M. 1984. Datos sobre la biologia reproductiva de Hoplostenum littorale (Siluriformes, Callichthyidae) de Venezuela. Acta Cient. Venez., Caracas, 35:142-146.

Mazzoni, R. \& Caramaschi, E. P. 1997. Spawning season, ovarian development and fecundity of Hypostomus affinis (Osteichthyes, Loricariidae). Revta bras. Biol., Rio de Janeiro, 57(3):455462 .

Nusssen, H. \& IsBrückeR, I. J. H. 1976. The South American plated catfish genus Aspidoras R. Von Ihering, 1907, with descriptions of nine new species from Brazil (Pisces, Siluriformes, Callichthyidae). Bijdr. Dierkd., Amsterdam, 46(1):107-131.

Nikolsky, G. V. 1963. The ecology of fishes. London, Academic. 352p.

REIS, R. E. 1998. Anatomy and phylogenetic analysis of the neotropical callicthyid catfishes (Ostariophysi, Siluriformes). Zool. J. Linn. Soc., London, 124(2):105-168.

Sokal, R. R. \& Rohlf, F. J. 1981. Biometry. New York, W. H. Freeman. 859p.

Strahler, A. N. 1957. Quantitative analysis of watershed geomorphology. Trans. Am. Geophys. Un., Washington, 38:913-920.

VAzzoler, A. E. A. DE M. 1981. Manual de métodos para estudos biológicos de populações de peixes: reprodução e crescimento. Brasília, CNPq-Programa Nacional de Zoologia. 108p.

1996. Biologia da reprodução de peixes teleósteos: teoria e prática. Maringá, EDUEM. $169 \mathrm{p}$.

Recebido em 29.01.2002 aceito em 30.09.2002.

Iheringia, Sér. Zool., Porto Alegre, 92 (4):89-98, 30 de dezembro 2002 